\title{
Rationale, design and methodology for Intraventricular Pressure Gradients Study: a novel approach for ventricular filling assessment in normal and falling hearts
}

Miguel Guerra ${ }^{1,2+}$, Mário J Amorim+, João C Mota², Luís Vouga ${ }^{2}$ and Adelino Leite-Moreira\# ${ }^{1,3^{*}}$

\begin{abstract}
Background: Intraventricular pressure gradients have been described between the base and the apex of the left ventricle during early diastolic ventricular filling, as well as, their increase after systolic and diastolic function improvement. Although, systolic gradients have also been observed, data are lacking on their magnitude and modulation during cardiac dysfunction. Furthermore, we know that segmental dysfunction interferes with the normal sequence of regional contraction and might be expected to alter the physiological intraventricular pressure gradients. The study hypothesis is that systolic and diastolic gradients, a marker of normal left ventricular function, may be related to physiological asynchrony between basal and apical myocardial segments and they can be attenuated, lost entirely, or even reversed when ventricular filling/emptying is impaired by regional acute ischemia or severe aortic stenosis.

Methods/Design: Animal Studies: Six rabbits will be completely instrumented to measuring apex to outflow-tract pressure gradient and apical and basal myocardial segments lengthening changes at basal, afterloaded and ischemic conditions. Afterload increase will be performed by abruptly narrowing or occluding the ascending aorta during the diastole and myocardial ischemia will be induced by left coronary artery ligation, after the first diagonal branch.

Patient Studies: Patients between 65-80 years old $(n=12)$, both genders, with severe aortic stenosis referred for aortic valve replacement will be selected as eligible subjects. A high-fidelity pressure-volume catheter will be positioned through the ascending aorta across the aortic valve to measure apical and outflow-tract pressure before and after aortic valve replacement with a bioprosthesis. Peak and average intraventricular pressure gradients will be recorded as apical minus outflow-tract pressure and calculated during all diastolic and systolic phases of cardiac cycle.

Discussion: We expect to validate the application of our method to obtain intraventricular pressure gradients in animals and patients and to promote a methodology to better understand the ventricular relaxation and filling and their correlation with systolic function.
\end{abstract}

\footnotetext{
* Correspondence: amoreira@med.up.pt

† Contributed equally

${ }^{1}$ Faculty of Medicine of University of Oporto, Department of Physiology,

Alameda Professor Hernâni Monteiro, 4202-451 Porto, Portugal

Full list of author information is available at the end of the article
} 


\section{Background}

Normal diastolic function of the left ventricle (LV) can be defined as the ability of the ventricle to adequately fill under low filling pressures. The hallmark of diastolic dysfunction is the impaired capacity to fill or maintain stroke volume without a compensatory increase in filling pressures [1,2]. Study of diastolic LV function should primarily be inspired by the impact that diastolic dysfunction has on symptoms and prognosis. Actually, diastolic dysfunction is present in a number of cardiac diseases and often precedes LV systolic dysfunction, leading to symptoms of heart failure in patients with preserved systolic function [3].

As early as 1930, Katz [4] already speculated that diastole was not entirely a passive process and the LV had the ability to "exert a sucking action to draw blood into its chamber." But it was only in 1979 that Ling et al. [5] first described, in a canine model, intraventricular pressure gradients (IVPG) during relaxation and filling of the LV. In 1988, Courtois et al. [6] observed, also in a canine model, a significant sub-basal-apical early diastolic pressure gradient along the LV inflow tract with minimum pressure in the apex speculating suction of the blood toward the LV apex. When subsequently it was shown that these gradients were diminished by ischemia and related to systolic function [7], the concept that they reflected recoil was born. Moreover, when Nikolic et al. [8] in 1995 demonstrated IVPG during early diastole in filling as well as in non-filling heart beats, the hope that IVPG would become an index for isovolumic and early ventricular relaxation was substantiated.

Therefore to describe LV diastolic function comprehensively, it is crucial the precise characterization of the transmitral and intraventricular pressure-flow relation. Early diastole is not amenable to analysis with simple passivefilling models, and any complete description of diastole must account for ventricular suction and for the presence of regional pressure oscillations which play an important role in normal ventricular filling [9]. In fact, the observation that the apical region fills first and begins to oscillate while filling is still occurring in the basal region is consistent with a model of diastolic function in which it can be inferred that suction is completed earlier near the apex than near the base [6,7]. Later [10], it was also demonstrated that in both animals and humans the pressure gradient between the ventricular apex and outflow tract strongly correlated with peak early transmitral flow and stroke volume and markedly increased during volume loading and decreased during reduced LV filling by caval constriction. Furthermore, in 2001, Firstenberg et al. [11] confirmed the existence of IVPG during early diastolic filling in humans and demonstrated that improvements in LV systolic and diastolic function, through surgical myocardial revascularization and/or LV remodeling, result in increases in IVPG. In fact, the same group has shown in patients with hypertrophic cardiomyopathy that diastolic IVPG are lower than in healthy subjects and improve after percutaneous septal ablation [12]. Although systolic IVPG has been also observed between the LV apex and the subaortic area [10], data are lacking on the magnitude of these gradients and its modulation during systolic and diastolic function impairment. Actually, regional ischemia interferes with the normal sequence of regional contraction and might be expected to alter the physiological diastolic and systolic IVPG.

These observations suggest the critical importance of IVPG to ensure efficient LV diastolic filling and allow us hypothesizing that any condition which interferes with the normal sequence of regional relaxation might be expected to change the physiological IVPG pattern. Actually, several studies have demonstrated that LV function is nonuniform in healthy hearts $[13,14]$. Peak shortening is larger in the lateral wall than in the septum and increases from the base to apex [15]. Besides variations in the degree of shortening, variations in the timing of shortening have been reported including early onset and late peak of shortening in the lateral wall [16]. Moreover, mechanical interaction between different myocardial segments has been studied extensively during regional ischemia, a condition in which regional myocardial function of the ischemic segment is decreased but that of the adjacent normal myocardium may be increased $[17,18]$. Understanding the origin of normal regional differences in LV myocardial function may give insight in pathological nonuniformities.

A variety of other disorders are associated with diastolic dysfunction, such as hypertrophy, structural alterations of the myocardium with increased fibrosis, myocardial scarring, or infiltrative processes [19]. In addition to these changes, physiological abnormalities of the LV with impaired relaxation, decreased diastolic filling, and increased stiffness of the myocardium can be observed [20]. In patients with aortic stenosis (AS), the most common cause for diastolic dysfunction is LV hypertrophy $[21,22]$. In subjects with asymptomatic severe AS, increased LV mass index was found to be an independent predictor for the development of symptoms [23]. Although it has been previously shown that aortic valve replacement (AVR) may lead to immediate hemodynamic improvement and to prolongation of survival $[24,25]$, it has been reported that regression of myocardial hypertrophy after relief of the hemodynamic burden is a process that may continue for decades after AVR [26]. However, abnormal exercise hemodynamics may persist late after AVR despite a normal systolic response [27], suggesting impaired diastolic function in these patients which may be revealed by acute and early IVPG alteration. 
Despite the apparent importance of IVPG in diastolic function evaluation, they have never been utilized in clinical cardiology, due to the complexity of their acquisition. Whereas regional pressure differences between the LV, the LV outflow tract, and the aorta during ejection have been recognized for some time [28], the importance of regional pressure differences within the ventricle during diastole and systole has only recently gained attention. Moreover, LV dysfunction may be underestimated when only LV ejection fraction is evaluated. Actually, tissue Doppler imaging [29] and 2dimensional strain [30] analysis of longitudinal myocardial function have shown to be superior in detecting subtle deteriorations of contractility. However, methodology to provide means for earlier diagnosis of global or regional myocardial disease remains an issue of study and necessary research [31-33].

In conclusion, we hypothesize that systolic and diastolic IVPG, a marker of normal left ventricular function, may be related to physiological asynchrony between basal and apical myocardial segments and that they can be attenuated, lost entirely, or even reversed when ventricular filling/emptying is impaired by acute regional ischemia or pressure overload, such as severe aortic stenosis.

\section{Objectives}

\section{Animal studies}

1) Characterize IVPG along the cardiac cycle (systole and diastole);

2) Evaluate the effects of the ischemia and modulation by afterload;

3) Correlate the IVPG with myocardial segmental asynchrony, in basal, afterloaded and ischemic conditions.

\section{Patient studies}

1) Validate the invasive measurement of IVPG for systolic and diastolic function evaluation in patients with severe AS;

2) Apply this methodology to evaluate whether the IVPG improve in AS patients immediately after AVR;

3) Establish if IVPG changes correlate with the reduction in LV obstruction and improvement in LV function;

4) Correlate catheter measurements with preoperative echocardiography;

5) Evaluate the potential clinical applicability of the concepts derived from the experimental studies.

\section{Methods and Design}

\section{Animal studies}

The investigation conforms to the Guide for the Care and Use of Laboratory Animals published by the US National Institutes of Health (NIH Publication No. 8523, Revised 1996).

\section{Animal preparation}

Male New Zealand White rabbits (Oryctolagus cuniculus, $\mathrm{n}=6)$ are premedicated with ketamine hydrochloride (50 $\mathrm{mg} / \mathrm{kg} \mathrm{im}$ ) and xylazine hydrochloride (5 mg/kg im). A femoral vein is cannulated, and a solution containing 20 meq $\mathrm{KCl}$ and 40 meq $\mathrm{NaHCO}_{3}$ in $500 \mathrm{ml}$ of $0.9 \%$ $\mathrm{NaCl}$ is administrated at a rate of $8 \mathrm{ml} \cdot \mathrm{kg}^{-1} \cdot \mathrm{h}^{-1}$ to compensate for perioperative fluid losses. A tracheostomy is performed, and mechanical ventilation is initiated (Harvard Small Animal Ventilator, model 683), delivering oxygen-enriched air. Respiratory rate and tidal volume are adjusted to keep arterial blood gases and $\mathrm{pH}$ within physiological limits. Anesthesia is maintained with a perfusion of midazolam $\left(0.07 \mathrm{mg} \cdot \mathrm{kg}^{-1} \cdot \mathrm{h}^{-1}\right)$, fentanil $(0.003$ $\left.\mathrm{mg} \cdot \mathrm{kg}^{-1} \cdot \mathrm{h}^{-1}\right)$ and vecuronium $\left(0.1 \mathrm{mg} \cdot \mathrm{kg}^{-1} \cdot \mathrm{h}^{-1}\right)$. A 20 gauge catheter is inserted in the right femoral artery and connected to a pressure transducer to monitor heart rate and arterial pressure and to obtain samples for blood gas analysis. The heart is exposed by a median sternotomy, and the pericardium is widely opened. One silk suture is placed around the ascending aorta and then passed through a plastic tube to perform transient aortic occlusions during the experimental protocol. A limb electrocardiogram (DII) is recorded throughout.

\section{Pressure measurements}

Two 3-F high-fidelity micromanometer (SPR-524, Millar Instruments, Houston, Tex., USA) are inserted through an apical puncture wound into the LV cavity. One is pulled carefully back toward the endocardium and secured in place with a purse-string suture to measure apical LVP. The other is introduced until we can see the impact from the aortic valve on the pressure trace. The catheter then is pulled back $5 \mathrm{~mm}$ below the aortic valve so that it is located in the $\mathrm{LV}$ outflow-tract to measure basal LVP. The pressure transducers are calibrated against a mercury column and zeroed after stabilization for $30 \mathrm{~min}$ in a water bath at body temperature. The zero is set at the level of the right atrium. Recordings are made with respiration suspended at end expiration. Parameters are converted on-line to digital data with a sampling frequency of $1 \mathrm{kHz}$. LV pressures are measured at end-diastole (LVPED), at pressure nadir $\left(\mathrm{LVP}_{\text {min }}\right)$ and at peak systole $\left(\mathrm{LVP}_{\text {max }}\right)$. Peak rates of $\mathrm{LV}$ pressure rise $\left(\mathrm{dP} / \mathrm{dt}_{\max }\right)$ and pressure fall $\left(\mathrm{dP} / \mathrm{dt}_{\min }\right)$, as well as, time to $\mathrm{dP} / \mathrm{dt}_{\min }$ are measured too. Relaxation rate are estimated with the time constant tau $(\tau)$ by fitting the isovolumetric pressure fall to a monoexponential function. We pretend to record continuously IVPG as apical minus outflow-tract LVP. Peak and average (area) IVPG are calculated during diastolic and systolic phases of cardiac cycle.

\section{Sonomicrometry}

Regional ventricular function is measured with two pairs of ultrasonic segment length gauges implanted in the 
circumferential direction of apical and basal left ventricular anterior midwall and connected to a sonomicrometer amplifier system (Triton Technology, San Diego, CA). At the end of the experiment, the animals are sacrificed with an overdose of anesthetics, and the position of the crystals and micromanometers are verified at necropsy. Segment lengths are measured at the end diastole (ED Length), at $\mathrm{dP} / \mathrm{dtmax}$ and at mitral valve opening (MVO). Minimum segment length (Lengthmin) was measured as the minimum length preceding or coinciding with peak $-\mathrm{dP} / \mathrm{dt}$. Fractional shortening was calculated as the percent segment length change from end diastole to $\mathrm{dP} / \mathrm{dtmin}$ at the outflow tract.

\section{Experimental protocol}

After complete instrumentation, we allow the animal preparation to stabilize for $30 \mathrm{~min}$ before the beginning of the experimental protocol. This consists in measuring apex to outflow-tract pressure gradient and apical and basal myocardial segments lengthening changes at basal, afterloaded and ischemic conditions.

\section{Afterload manipulation}

Sudden afterload elevations are performed by abruptly narrowing or occluding the ascending aorta during the diastole, as previously described [34,35]. In summary, this is achieved by pushing the plastic tube against the aorta with one hand while pulling the silk suture with the other hand. The analyzed intervention, therefore, is a selective alteration of afterload without changes of preload or long-term load history. The aortic clamp is quickly released to avoid neurohumoral reflex changes in cardiac function. The animal is stabilized for several beats before another intervention is performed.

\section{Myocardial ischemia induction}

Myocardial ischemia is induced by left coronary artery (LCA) ligation, after the first diagonal branch. Visible collateral arteries are tied as well to induce an anteroapical ischemia. Recordings are performed after $30 \mathrm{~min}$. Mortality is documented.

\section{Patient studies}

Full ethical approval for this study has been obtained from Ethics Committee of Centro Hospitalar de Vila Nova de Gaia, EPE. It is conducted in accordance with the principles of The Declaration of Helsinki, with the Portuguese laws and rules and subscribes to the principles outlined in the International Conference on Harmonisation of Good Clinical Practice [36].

All patients receive full explanation of study objectives, the operations to be performed, its risks and benefits and signed the informed consent form.

Any death or major complication during the study period requires the hospital ethical commission to be informed.

\section{Study population}

Patients between 65-80 years old $(\mathrm{n}=12)$, both genders, with severe aortic stenosis (aortic valve area $[\mathrm{AVA}]<$ $1.0 \mathrm{~cm}^{2}$ ) referred for aortic valve replacement (AVR) are selected as eligible patients. Patients with any one of the following are excluded from the study: concomitant severe mitral regurgitation; mitral stenosis, regardless of severity; any prosthetic heart valve; coronary artery disease; concomitant aortic regurgitation; a history of surgical or percutaneous aortic valvuloplasty; history of ethanol abuse; and chronic obstructive pulmonary disease that are worse than mild as assessed clinically and/ or confirmed by pulmonary function testing. (Table 1) The baseline and follow-up clinical variables and pharmacological data are obtained from a review of the medical records.

\section{Intraoperative procedure}

All patients undergo routine induction of general anesthesia, median sternotomy, and pericardiotomy. After great vessel cannulation and systemic heparinization, a high-fidelity pressure-volume catheter (Cardiovascular Millar Mikro-Tip ${ }^{\circledR}$ ) is positioned from a small ascending aorta stab incision across the aortic valve. The 2 pressure sensors are positioned in the LV cavity in apex and in outflow-tract (sub-aortic valve) position. Appropriate anatomic placement is confirmed through the use of transesophageal echocardiography and visualization of appropriate chamber-specific waveforms.

\section{Hemodinamic measurements}

For each patient, during suspended ventilation, recordings of intracardiac pressure-volumes are obtained before cardiopulmonary bypass (CPB) beginning. After adequate data collection, the catheter is removed and placed in warm saline, myocardial arrest with full CPB support is obtained, and each patient undergoes

\section{Table 1 Patient Eligibility Criteria}

\begin{tabular}{ll}
\hline Inclusion & Exclusion \\
\hline Symptomatic aortic valve & Concomitant $>$ mild mitral regurgitation \\
Aortic valve area $<1.0 \mathrm{~cm}^{2}$ & Mitral stenosis regardless of severity \\
First time cardiac surgery & Concomitant aortic regurgitation \\
Age 65-80 years old & Angiographic coronary artery disease \\
Signed informed consent & Chronic atrial fibrillation \\
& History of percutaneous aortic \\
& valvuloplasty \\
& History of ethanol abuse \\
& Chronic obstructive pulmonary disease \\
& $>$ mild \\
& Urgent or emergent surgery \\
& Associated surgical procedure \\
& Creatinin $>1.5$ ULN \\
& Inability to give informed consent
\end{tabular}


biological AVR. After completely weaned from CPB and volume infusions from the $\mathrm{CPB}$ circuit to obtain adequate hemodynamics by increasing preload, after rezeroing, the catheter is repositioned across the aortic valve, and multiple hemodynamic measurements are obtained in several intervals during different stages of physiological stabilization. To compared with pre- $\mathrm{CPB}$ measurements catheters are matched at same LV end-diastolic pressure. During this period of data collection, no patient should require vasopressor, inotropic, or external pacing support. After data collection, the catheter is removed, systemic heparinization is reversed, and the operative procedure is concluded in the conventional fashion.

\section{Echocardiography}

All patients will have standard two-dimensional echocardiographic examinations before and after AVR. LV ejection fraction is assessed visually by a trained echocardiographer and entered into a database at the time of the examination. Anatomic and Doppler examinations and measurements are performed according to the recommendations of the American Society of Echocardiography. The aortic valve area is calculated using the continuity equation utilizing flow velocities in the LV outflow tract and across the valve. The pulmonary artery systolic pressure is calculated from the tricuspid regurgitation velocity signal using the simplified Bernoulli equation and estimated right atrial pressure based on inferior vena caval size. Doppler flow data is acquired from the LV outflow tract region in pulsed wave mode and from the aortic valve in continuous wave mode in the 5-chamber view. Peak velocities, calculated with resident software at the time of imaging, is used to calculate pressure gradients according to the modified Bernoulli equations and valve orifice areas according to the continuity equation approach.

\section{Statistical analysis}

All analyses are performed using SPSS statistical software (SPSS 17.0, Chicago, IL).

\section{Animal studies}

Group data are presented as means \pm SE and are compared using two-way ANOVA. Student-Newman-Keuls test is selected to perform pairwise multiple comparisons when significant differences are detected.

\section{Patient studies}

A two-tailed paired $t$ test is used to compare patients before and after AVR. Differences are considered statistically significant at $\mathrm{P}<0.05$.

\section{Discussion}

We expect 1) to validate the application of our invasive method to characterize the IVPG along cardiac cycle in physiological and pathological conditions; 2) to find a correlation between AS severity, diastolic dysfunction and IVPG impairment; 3) to provide new insights into the mechanical adaptation of LV to chronic afterload elevation and its response to unloading after AVR; 4) to show if the degree of hypertrophy parallels the severity of overload and if the assessment of IVPG can identify subtle contractile dysfunction; and 5) to promote the use of IVPG in clinical practice as another index of diastolic function and ventricular filling.

\section{Abbreviations}

LV: left ventricle; IVPG: intraventricular pressure gradients; AS: aortic stenosis; AVR: aortic valve replacement; LVPED: left ventricular pressure at enddiastole; $L V P_{\text {min }}$ : left ventricular pressure nadir; $L V P_{\text {max }}$ : left ventricular pressure at peak systole; $\mathrm{dP} / \mathrm{dt}_{\max }$ : peak rates of left ventricular pressure rise; $\mathrm{dP} / \mathrm{dt}_{\text {min }}$ : peak rates of left ventricular pressure fall; $\tau$ : time constant tau; ED Length: segment length at the end diastole; MVO: mitral valve opening; Length $_{\text {min }}$ : minimum segment length; LCA: left coronary artery; AVA: aortic valve area; CPB: cardiopulmonary bypass.

\section{Author details}

${ }^{1}$ Faculty of Medicine of University of Oporto, Department of Physiology, Alameda Professor Hernâni Monteiro, 4202-451 Porto, Portugal. ${ }^{2}$ Centro Hospitalar de Vila Nova de Gaia/Espinho, EPE, Department of Cardiothoracic Surgery, Rua Conceição Fernandes, 4434-502 Vila Nova de Gaia, Portugal. ${ }^{3}$ Hospital de São João, Department of Cardiothoracic Surgery, Alameda Professor Hernâni Monteiro, 4202-451 Porto, Portugal.

\section{Authors' contributions}

MG and MJA contributed equally to this work. MG, MJA, JCM and ALM conceived and designed the protocol. MG and ALM contributed to the draft and final version of the manuscript. ALM and LV supervised the research project. All authors have read and approved the final manuscript.

\section{Declaration of competing interests}

The authors declare that they have no competing interests.

Received: 15 January 2011 Accepted: 10 May 2011

Published: 10 May 2011

\section{References}

1. Vasan RS, Levy D: Defining diastolic heart failure: a call for standardized diagnostic criteria. Circulation 2000, 101:2118-2121.

2. Leite-Moreira AF: Current perspectives in diastolic dysfunction and diastolic heart failure. Heart 2006, 92(5):712-718.

3. Vasan RS, Benjamin EJ, Levy D: Prevalence, clinical features and prognosis of diastolic heart failure: an epidemiologic perspective. J Am Coll Cardiol 1995, 26:1565-1574.

4. Katz $L N$ : The role played by the ventricular relaxation process in filling the ventricle. Am J Physiol 1930, 95:542-553.

5. Ling D, Rankin JS, Edwards CH II, McHale PA, Anderson RW: Regional diastolic mechanics of the left entricle in the conscious dog. Am J Physiol 1979, 236:H323-330.

6. Courtois M, Kovacs SJ, Ludbrook PA: Transmitral pressure-flow velocity relation: importance of regional pressure gradients in the LV during diastole. Circulation 1988, 78:661-671

7. Courtois MA, Kovacs SJ, Ludbrook PA: Physiologic early diastolic intraventricular gradient is lost during acute myocardial ischemia. Circulation 1990, 81:1688-1696.

8. Nikolic SD, Feneley MP, Pajaro OE, Rankin JS, Yellin EL: Origin of regional pressure gradients in the LV during early diastole. Am J Physio/ 1995, 268 H550-557.

9. Pasipoularides A, Mirsky I, Hess OM, Grimm J, Krayenbuehl : Myocardial relaxation and passive diastolic properties in man. Circulation 1986, 74:991-1001

10. Smiseth OA, Steine K, Sandbaek G, Stugaard M, Gjolberg T: Mechanics of intraventricular filling: study of LV early diastolic pressure gradients and flow velocities. Am J Physiol 1998, 275:H1062-1069. 
11. Firstenberg MS, Smedira NG, Greenberg NL, Prior DL, McCarthy PM, Garcia MJ, Thomas JD: Relationship between early diastolic intraventricular pressure gradients, an index of elastic recoil, and improvements in systolic and diastolic function. Circulation 2001, 104(Suppl I):330-335

12. Rovner A, Smith R, Greenberg NL, Tuzcu EM, Smedira N, Lever HM, Thomas JD, Garcia MJ: Improvement in diastolic intraventricular pressure gradients in patients with HOCM after ethanol septal reduction. Am J Physiol Heart Circ Physiol 2003, 285:H2492-2499.

13. Leite-Moreira AF, Gillebert TC: Nonuniform course of left ventricular pressure fall and its regulation by load and contractile state. Circulation 1994, 90:2481-2491.

14. Bogaert J, Rademakers FE: Regional nonuniformity of normal adult human left ventricle. Am J Physiol Heart Circ Physiol 2001, 280:H610-620

15. Moore CC, Lugo-Olivieri CH, McVeigh ER, Zerhouni EA: Three dimensional systolic strain patterns in the normal human left ventricle: characterization with tagged MR imaging. Radiology 2000, 214:453-466

16. Zwanenburg JJM, Gotte MJW, Kuijer JPA, Heethaar RM, Van Rossum AC Marcus JT: Timing of cardiac contraction in humans mapped by hightemporal resolution MRI tagging: early onset and late peak of shortening in the lateral wall. Am J Physiol Heart Circ Physiol 2004, 286 : H1872-1880.

17. Lew WY, Chen ZY, Guth B, Covell JW: Mechanisms of augmented segment shortening in nonischemic areas during acute ischemia of the canine left ventricle. Circ Res 1985, 56:351-358.

18. Smalling RW, Ekas RD, Felli PR, Binion L, Desmond J: Reciprocal functional interaction of adjacent myocardial segments during regional ischemia: an intraventricular loading phenomenon affecting apparent regional contractile function in the intact heart. J Am Coll Cardiol 1986, 7:1335-1346

19. Hess OM, Villari B, Krayenbuehl HP: Diastolic dysfunction in aortic stenosis. Circulation 1993, 87(5 Suppl):IV73-76.

20. Gillebert TC, Leite-Moreira AF, De Hert SG: Load dependent diastolic dysfunction in heart failure. Heart Fail Rev 2000, 5(4):345-355.

21. Lavine SJ, Follansbee WP, Schreiner DP, Amidi M: Left ventricular diastolic filling in valvular aortic stenosis. Am J Cardiol 1986, 57:1349-1355.

22. Steine $K$, Rossebø AB, Stugaard M, Pedersen TR: Left ventricular systolic and diastolic function in asymptomatic patients with moderate aortic stenosis. Am J Cardiol 2008, 102(7):897-901.

23. Dinh W, Nickl W, Smettan J, Kramer F, Krahn T, Scheffold T, Barroso MC, Brinkmann H, Koehler T, Lankisch M, Füth R: Reduced global longitudinal strain in association to increased left ventricular mass in patients with aortic valve stenosis and normal ejection fraction: a hybrid study combining echocardiography and magnetic resonance imaging. Cardiovasc Ultrasound 2010, 8:29.

24. Toussaint C, Cribier A, Cazor UL, Soyer R, Letac B: Hemodynamic and angiographic evaluation of aortic regurgitation 8 and 27 months after aortic valve replacement. Circulation 1981, 64(3):456-463.

25. Schwarz F, Baumann P, Manthey J, Hoffmann M, Schuler G, Mehmel HC, Schmitz W, Kübler W: The effect of aortic valve replacement on survival. Circulation 1982, 66(5):1105-1110.

26. Carroll JD, Gaasch WH, Naimi S, Levine HJ: Regression of myocardial hypertrophy: electrocardiographic-echocardiographic correlations after aortic valve replacement in patients with chronic aortic regurgitation. Circulation 1982, 65(5):980-987.

27. Monrad ES, Hess OM, Murakami T, Nonogi H, Corin WJ, Krayenbuehl HP. Abnormal exercise hemodynamics in patients with normal systolic function late after aortic valve replacement. Circulation 1988, 77(3):613-624

28. Lombard JT, Selzer A: Valvular aortic stenosis. A clinical and hemodynamic profile of patients. Ann Intern Med 1987, 106(2):292-298

29. Abraham TP, Dimaano VL, Liang HY: Role of tissue Doppler and strain echocardiography in current clinical practice. Circulation 2007, 116:2597-2609.

30. Galema TW, Yap SC, Geleijnse ML, van Thiel RJ, Lindemans J, ten Cate FJ, Roos-Hesselink JW, Bogers AJ, Simoons ML: Early detection of left ventricular dysfunction by Doppler tissue imaging and $\mathrm{N}$-terminal proBtype natriuretic peptide in patients with symptomatic severe aortic stenosis. J Am Soc Echocardiogr 2008, 21:257-261.

31. Mendoza DD, Codella NCF, Wang Y, Prince MR, Sethi S, Manoushagian SJ, Kawaji K, Min JK, LaBounty TM, Devereux RB, Weinsaft JW: Impact of diastolic dysfunction severity on global left ventricular volumetric filling - assessment by automated segmentation of routine cine cardiovascular magnetic resonance. Journal of Cardiovascular Magnetic Resonance 2010, 12:46.

32. Licker M, Cikirikcioglu M, Inan C, Cartier V, Kalangos A, Theologou T, Cassina T, Diaper J: Preoperative diastolic function predicts the onset of left ventricular dysfunction following aortic valve replacement in highrisk patients with aortic stenosis. Critical Care 2010, 14:R101.

33. Apostolakis EE, Baikoussis NG, Parissis H, Siminelakis SN, Papadopoulos GS: Left ventricular diastolic dysfunction of the cardiac surgery patient; a point of view for the cardiac surgeon and cardio-anesthesiologist. Journal of Cardiothoracic Surgery 2009, 4:67.

34. Leite-Moreira AF, Correia-Pinto J, Gillebert TC: Afterload induced changes in myocardial relaxation: a mechanism for diastolic dysfunction. Cardiovasc Res 1999, 43(2):344-353.

35. Leite-Moreira AF, Correia-Pinto J: Load as an acute determinant of enddiastolic pressure-volume relation. Am J Physiol Heart Circ Physiol 2001, 280(1):H51-59.

36. "Guideline for Good Clinical Practice", ICH Tripartite Guideline. 2002.

doi:10.1186/1749-8090-6-67

Cite this article as: Guerra et al:: Rationale, design and methodology for Intraventricular Pressure Gradients Study: a novel approach for ventricular filling assessment in normal and falling hearts. Journal of Cardiothoracic Surgery 2011 6:67.

\section{Submit your next manuscript to BioMed Central and take full advantage of:}

- Convenient online submission

- Thorough peer review

- No space constraints or color figure charges

- Immediate publication on acceptance

- Inclusion in PubMed, CAS, Scopus and Google Scholar

- Research which is freely available for redistribution

Submit your manuscript at www.biomedcentral.com/submit
C Biomed Central 